Mátyás Gede, Valentin Árvai, Gergely Vassányi, Zsófia Supka, Enikő Szabó, Anna Bordács, Csaba Gergely Varga, Krisztina Irás ${ }^{1}$

\title{
Automatic vectorisation of old maps using QGIS - tools, possibilities and challenges
}

Keywords: map vectorisation, QGIS, Python

Summary: The authors experimented with the open source GIS software QGIS to reveal its possibilities in automatic vectorisation. The target object was a $1: 200000$ map sheet of the third Austro-Hungarian military survey. Although QGIS provides tools for the main steps of the vectorisation process - such as colour segmentation of the raster image, skeletoning, vector extraction and vector post-processing using them on an old map raises several challenges. This paper discusses the results of these experiments, and introduces a few simple python scripts that may help users in the vectorisation process.

\section{Introduction}

Historical maps are an important source of topographic, statistical and other types of information of past ages. Although it is possible to manually extract the required data from these maps, it is a tedious process especially in the case of sheets of old surveys that have very high information density. The goal is usually to transform specific content of these maps into vectorbased feature data with attributes that can be further processed, analysed by GIS software. There are various attempts to fasten this process by automatizing certain steps or, if possible, the whole task.

Brown (2002) uses the commercial R2V software for vectorising geological maps. Arteaga (2013) developed the R tool Map Vectorizer, optimized for building outline extraction from cadastral/insurance maps. Iosifescu et al. (2016) experimented on $19^{\text {th }}$ and early $20^{\text {th }}$ century Swiss topographic maps also focusing mostly on building footprints but dealing with hydrography as well. They used various open source software: GDAL, ImageMagick and QGIS with GRASS. Peller (2018) provides a comprehensive review of several projects on this field, utilizing commercial or open source solutions.

Commercial GIS software ArcGIS provides the extension ArcScan to help vectorisation process. It is able to trace lines, recognise basic geometric shapes on raster images (ESRI, 2016). Open source QGIS also has modules for the task but these are not (yet) organized into a single user-friendly tool. Automatic vectorisation algorithms are often used on satellite imagery for extracting linear features (e.g. rivers) or landcover polygons (e.g. in Schwenk et al, 2018). All vectorisation methods follow the same chain of steps (although usually not implementing all of them):

- Scanning,

- Georeferencing,

- Image pre-processing (colour enhancement, noise removal, etc.),

\footnotetext{
${ }^{1}$ Department of Cartography and Geoinformatics, ELTE Eötvös Loránd University, Budapest [saman@map.elte.hu]
} 
- Colour segmentation,

- Raster editing (line thinning, removing small objects etc.),

- Raster-vector conversion,

- Vector post-processing.

While works mentioned above mainly deal with large scale (mostly cadastral) maps, this paper summarizes the results of vectorisation experiments on late $19^{\text {th }}$ and early $20^{\text {th }}$ century topographic maps, mostly in QGIS environment.

\section{Target object}

The target object (Figure 1) of the present study is a $1: 200000$ (Generalkarte) map sheet of the third Austro-Hungarian military survey depicting the confluence of river Olt into the Danube. The coverage of this map series stretches far beyond the actual territory of AustriaHungary. This sheet was chosen because it is in a very good state comparing to other sheets physically available at our department and it depicts several various landscape types which makes it perfect for testing vectorisation methods.

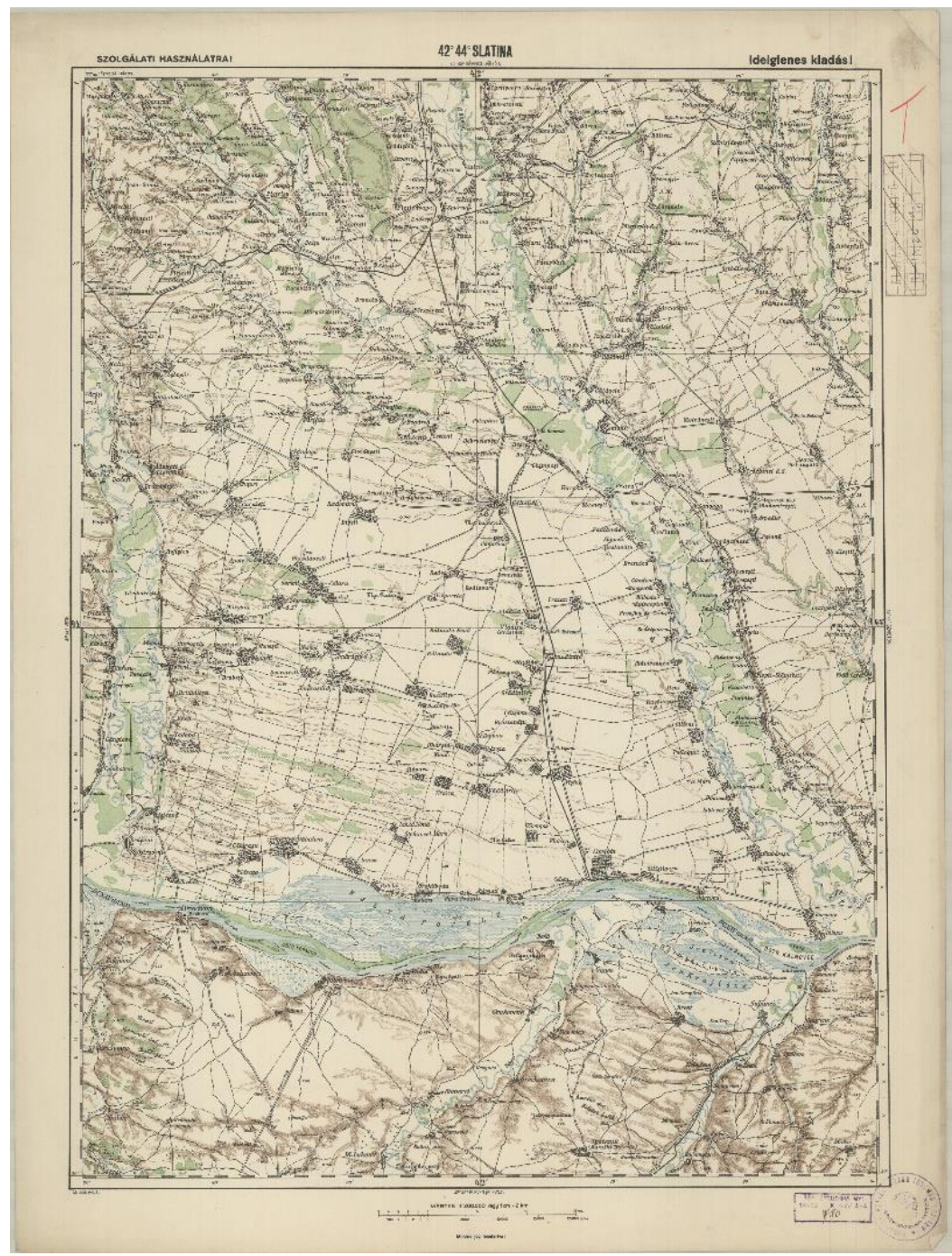

Figure 1. " $42^{\circ} 44^{\circ}$ Slatina" sheet, the target object of present study. (Courtesy of the Map Collection of ELTE Eötvös Loránd University) 


\section{Processing steps}

\section{Scanning}

Although the first step, scanning the maps seems to be a quite straightforward thing, choice of resolution, colour depth, and image format may affect the success of every following step. If the resolution is too low, important details can be lost and nearby objects can be blurred. On the other hand, using too high resolution, the material errors and print errors of the paper (for example smudged ink) might also appear after vectorisation. The sample map sheet was scanned in $600 \mathrm{dpi}$, as a 24 bit colour TIFF image with lossless compression. This latter is important - using a JPEG compression on the image causes loss in the original pixel information.

\section{Georeferencing}

The Generalkarte sheets of the $3^{\text {rd }}$ Military Survey were composed in the Lichtenstern polyhedric projection (Timár et al, 2009). As this projection is not supported by any known GIS software, a projection with similar properties is required. The Lichtenstern projection maps the selected $1^{\circ}$ by $1^{\circ}$ geographic quadrangle into a trapezium in which the central meridian as well as the bounding latitudes are true scale, so applying the Sinusoidal projection on it with proper datum and central meridian settings will result infinitesimal distortion as this projection also has true scale central meridian and parallels, and the bend of the edge meridians of the $1^{\circ}$ by $1^{\circ}$ quadrangle is negligible at this latitude. Although the georeferencing process also can be carried out in QGIS, the authors used Global Mapper for this task, using the corners and the edge midpoints as ground control points (GCPs).

\section{Image preprocessing}

Depending on the type of features to be extracted, there might be different image preprocessing solutions needed. The current map depicts water bodies and larger rivers with a horizontal blue hatch fill. This pattern usually needs to be blurred into a uniform colour before vectorising, which can be realized using a convolutional filter with cross-shaped kernel matrix. If the goal is the enhancement of these horizontal lines, another kernel with a horizontal line can be used (Figure 2 and 3). This preprocessing can be done for example in GIMP (to stay on the open source field).

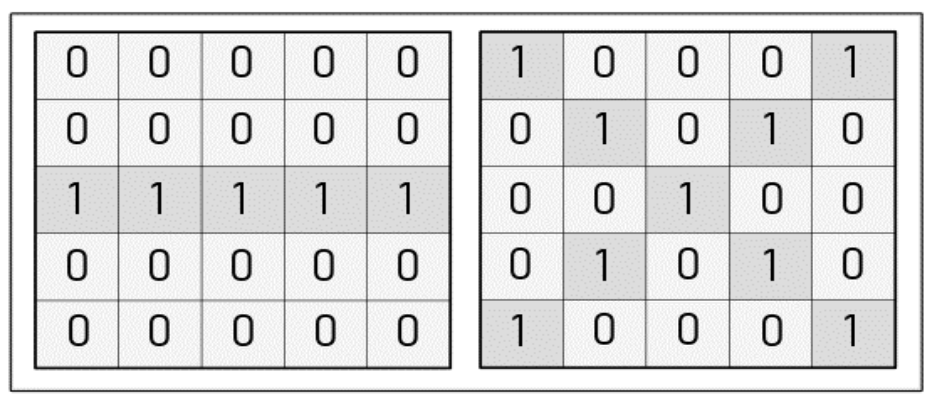

Figure 2. Kernel matrices for enhancing (left) or blurring (right) horizontal hatching 


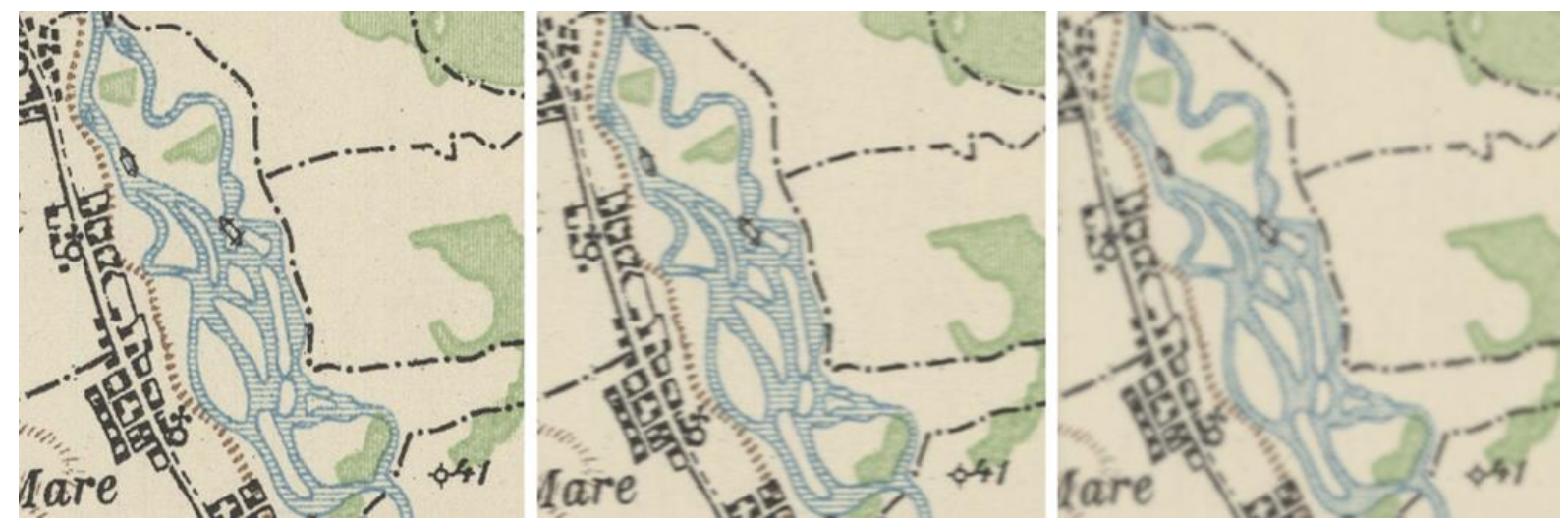

Figure 3. The original image with enhanced (left) and blurred (right) hatch

\section{Colour segmentation}

Before vectorising, we need to select those pixels of the raster that the features are shown by. This is called colour segmentation and depending on the method used may be resulted in a binary raster (where pixel values are set to 1 or 0 based on their colour belongs to that given feature type or not) or to a palette image in which there are a handful of possible colour values corresponding to the original printing colours of the map.

The dzetsaka plugin of QGIS does the latter. The user needs to create a few polygons on a vector layer, marking examples of the various printing colours (classes), and the plugin uses this information to classify pixels (Karasiak, 2016). This plugin proved to be successful for extracting black features (roads, buildings, labels). For rivers, however, another solution was used, taking advantage that blue was overprinted on other colours, therefore, by converting the image into HSV (hue/saturation/value) colour model and using hue filtering the blue features can be extracted even when they are printed upon other colours.

\section{Raster editing}

Due to the outworn paper of archive maps, the faded colours on it and eventual scanning errors, colour segmentation usually results in a raster that needs further processing before vectorisation: removal of small holes, standalone pixels, which is usually followed by skeletoning (thinning) lines. In QGIS, there are many ways for that. SAGA Close One Cell Gaps module fills every 1-pixel hole and makes the objects more homogeneous. With Remove small pixel clumps function the values of lone pixels, small pixel clumps can be changed to "no data" and after that these pixels are not in use. GDAL Translate (convert format) function can convert the pixels with 0 value (which means unwanted background colours) to "no data".

To help colour segmentation and raster editing task, a simple Python application was developed (see scripts created for the project: https://github.com/samanbey/auto_vector). The program is based on OpenCV and the rivgraph module (Schwenk, 2020).

The interface of HSV filter program (Figure 4) consists of a set of sliders. The first six slider of interface can be used to define the limit of the HSV values of highlighted colour. There are more six sliders to eliminate the inaccuracy derived from errors of paper or scanning by setting properties of the erosion filter kernel, the gradient filter and the closing filters. The erosion filter eliminates remove small pixel clumps and makes lines thinner, the gradient filter can be used to extract outlines while the closing filter fills small gaps. 

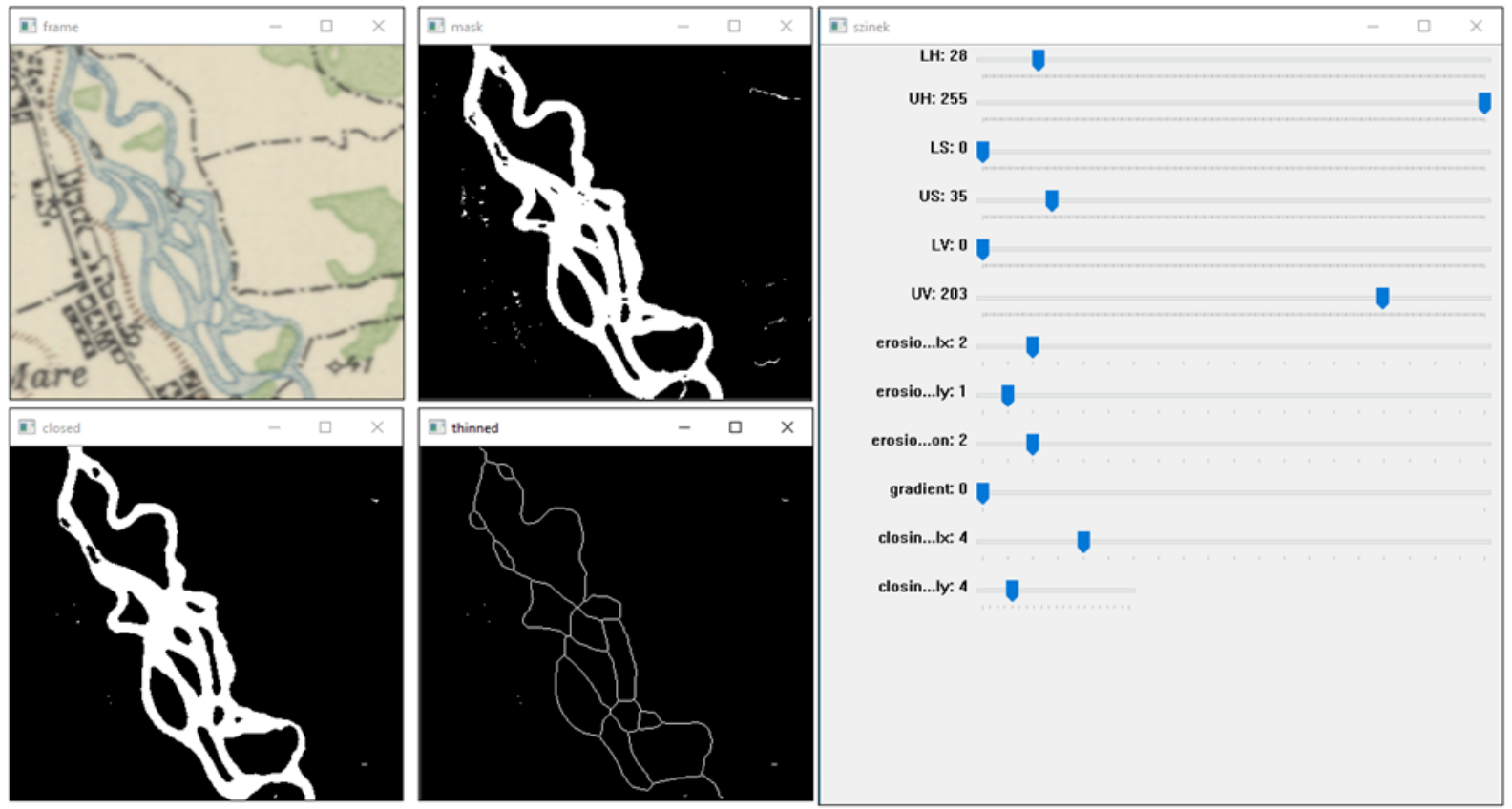

Figure 4. User interface and results of the colour filtering/skeletoning program

Another approach to thinning lines is using the "r.thin" function in the GRASS module of QGIS. Thinning is a crucial step before line vectorisation as the conversion only works with one-pixel wide lines.

\section{Vectorising lines and vector post-processing}

The thinned raster can be converted to vector using the "r.to.vect" function from GRASS module. The resulting set of polylines are still far from good: most lines are disjointed or branched into spiky short sections. The first step to eliminate these is running "v.clean" function with rmdangle command in GRASS module to remove most of the spikes. Some small branches still remain around the lines, so in SAGA module with "line properties" function is used to insert line segment lengths into the attribute table after which lines shorter than a certain limit are deleted from the table (The limit should be slightly larger than the actual pixel size of the raster). Finally, a geometric reduction is performed using the "Simplify" function in Vector/Geometry Tools menu.

Line vectorisation can also be carried out without thinning. In this case "r.to.vect" is used to create polygons from the raster image. Lines will be represented as polygon stripes. In the next step the holes inside the polygons are removed with "delete holes" function and a geometric reduction is performed with the "Simplify" function from Vector/Geometry Tools menu. Then the centerlines of polygons are created by the "Skeleton/Medial axis" function in $H C M G I S$ plugin in Geometry Processing menu. To remove the remaining spikes and branches, the "v.clean" function is used.

The only remaining task is joining the still separated line segments. A simple python script can do the trick in QGIS. The algorithm iterates over possible polyline pairs and checks the distance of their endpoints. If it is below a given threshold and the orientation of the corresponding line segments is also similar, the lines are joined. The algorithm continues until no more line joins are performed. See script at: https://github.com/samanbey/auto_vector 


\section{Results}

We got the best results so far when extracted rivers depicted as surfaces (with horizontal hatch fill) either as vector surfaces or generalized into polylines (Figure 5). Results with single-line hydrography elements (creeks, smaller rivers) are also promising, but auto-connecting fragments are not always perfect. Another problem with this category that colour filtering settings depend on how other map elements interfere with hydrography.

The toughest case is in mountainous areas where forest patches and relief hatching both may intersect water, not mentioning roads in narrow valleys running very close to creeks, and crossing them several times. Figure 6 shows results in such a sample area. Although most of the hydrographic network is well extracted, the resulting polylines are fragmented. Automatic connection of these lines did not bring the expected results yet; depending on the parameter settings many gaps remained unconnected or the script also connected line ending that should not be connected. Road and railroad network is also troublesome, mainly because the same black colour is used for several different element groups: Buildings, fences, roads, tracks, railways and place names.

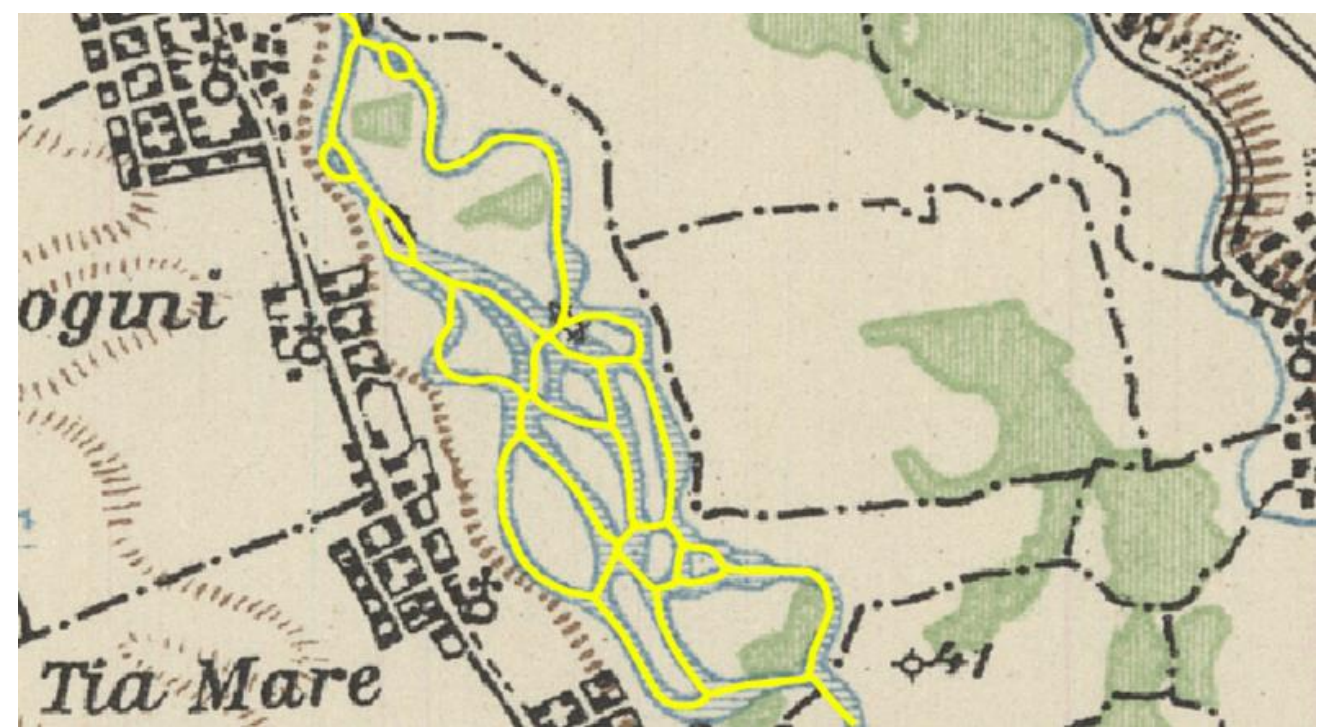

Figure 5. Rivers extracted as polylines

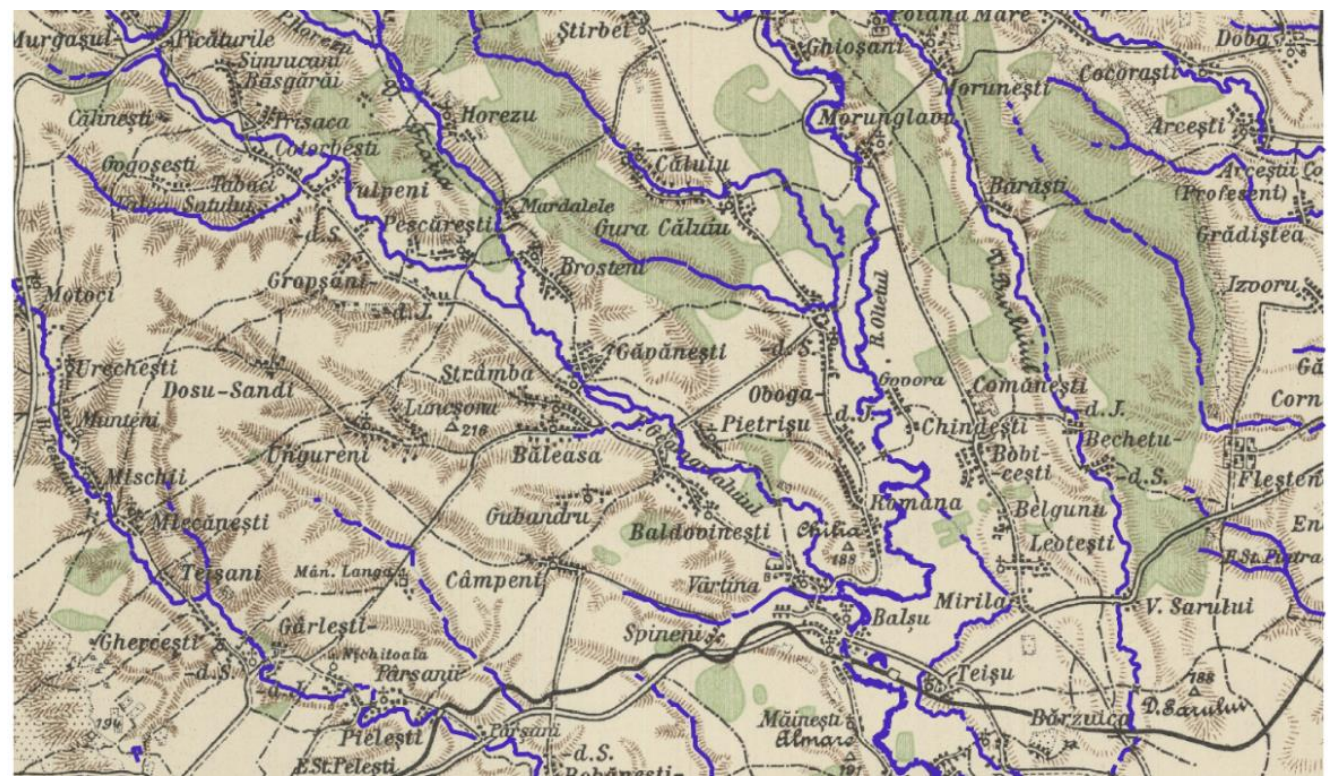

Figure 6. Recognition of single-line rivers in mountainous environment 


\section{Conclusion, further plans}

Results show that it is possible to automatically extract vector features from scanned old maps using QGIS, but there is no uniform solution for all feature types. The steps of extraction depend on printing colours, the interference of various map elements and the content density of the map. The results are still far from prefect, and further improvements are needed.

Future plans include the improvement of vector post processing - especially auto-connecting fragmented lines, recognising linear elements with double or dashed lines. Another field of interest is recognising and extracting map symbols and labels - this may also help in distinguishing different element categories printed in the same colour, as the already recognised elements can be removed from the raster image before further processing.

\section{Acknowledgement}

EFOP-3.6.3-VEKOP-16-2017-00001: Talent Management in Autonomous Vehicle Control Technologies. The Project is supported by the Hungarian Government and co-financed by the European Social Fund.

\section{References}

Arteaga, M. (2013). Historical Map Polygon and Feature Extractor. Proceedings of the 1st ACM SIGSPATIAL International Workshop on MapInteraction. New York, NY: ACM, pp. 66-71. https://doi.org/10.1145/2534931.2534932

Brown, K. (2002). Raster to Vector Conversion of Geologic Maps: Using R2V from Able Software Corporation. USGS Open-File Report 02-370. https://pubs.usgs.gov/of/2002/of02370/brown.html

ESRI. (2016). Getting Started with ArcScan: ArcMap 10.4. http://desktop.arcgis.com/en/arcmap/10.4/extensions/arcscan/what-is-arcscan-.htm Iosifescu, I., A. Tsorlini, L. Hurni (2016). Towards a comprehensive methodology for automatic vectorization of raster historical maps. e-Perimetron, Vol. 11, No.2, 2016 [57-76]

Karasiak, N. (2016) Dzetsaka: classification tool. https://github.com/nkarasiak/dzetsaka Peller, P. (2018) From Paper Map to Geospatial Vector Layer: Demystifying the Process, IASSIST Quarterly 42 (3), pp. 1-22. https://doi.org/10.29173/iq914

Schwenk, J., A. Tejedor, A Piliouras, E Foufoula-Georgiou, J Rowland, (2018). Automatic Extraction of Channel Network Topology (AGU 2018)

Schwenk, J. (2020). RivGraph. https://github.com/jonschwenk/RivGraph

Timár, G., G. Molnár, V. Crăciunescu, (2009). Georeference of the 1:200,000 'degree maps' of Central Europe (about 1910). Geophysical Research Abstracts, Vol. 11, EGU2009-2574, 2009 